\title{
la toma de decisiones. Una revisión del tema
}

\section{Decisionmaking. A review of the topic}

\author{
José Arévalo Ascanio ${ }^{1}$
} Hilda Estrada López ${ }^{2}$

* Este artículo constituye un avance del proyecto de investigación "La toma de decisiones. Estudio comparado entre empresarios exitosos del municipio de Ocaña".

1 Universidad Francisco de Paula Santander, Ocaña, Norte de Santander jgarevaloa@ufpso.edu.co - https://orcid.org/0000-0001-5464-2293

2 Universidad del Atlántico. Barranquilla-Colombia. hileslo@hotmail.com - https://orcid.org/0000-0001-7439-2658 


\title{
Gerencia de las organizaciones. \\ Un enfoque empresarial
}

\section{RESUMEN}

La toma de decisiones es un acto propio de nuestra vida. A cada momento se nos presenta un abanico de opciones, entre las cuales como personas debemos establecer una elección. Por tanto, este evento se reconoce como una línea de trabajo de alto impacto sobre las personas, las organizaciones y la sociedad en general. El objetivo de este artículo fue analizar la toma de decisiones desde una revisión teórica. El documento se divide en tres partes. En la primera se hace una aproximación al concepto de decisión. En la segunda, se revisan los documentos teóricos hallados sobre la temática. En la tercera parte se presenta el análisis de los trabajos empíricos que se han desarrollado sobre el tema por parte de la comunidad científica. Se finaliza, con las reflexiones en las que se hace hincapié en la utilidad del artículo,

250 al compartir un marco de referencia conceptual y empírico sobre la toma de decisiones en el contexto empresarial.

Palabras clave: decisión, decisiones gerenciales, toma de decisiones.

\begin{abstract}
Decision making is an act of our life. At each moment we are presented with a range of options, among which as people we must establish a choice. Therefore, this event is recognized as a line of work of high impact on people, organizations and society in general. The objective of this article was to analyze the decision making from a theoretical review. The document is divided into three parts. In the first, an approach to the concept of decision is made. In the second, the theoretical documents found on the
\end{abstract}


subject are reviewed. In the third part, the analysis of the empirical works that have been developed on the subject by the scientific community is presented. Finally, with the reflections emphasizing the usefulness of the article, by sharing a conceptual and empirical frame of reference on decision making in the business context.

Keywords: decision, management decisions, decision making.

\section{INTRODUCCIÓN}

Este trabajo pretende ilustrar el tema de la toma de decisiones a partir de una revisión bibliográfica en libros de texto y en revistas de alto impacto publicados en las bases de datos SCOPUS, SCIENCE DIRECT, EBSCO, SciELO y REDALYC. La estructura y el contenido de este documento son una muestra de los diferentes estudios, tanto teóricos como empíricos, existentes en la literatura. La toma de decisiones es uno de los temas de mayor trascendencia en la teoría organizacional, ya que, de modo evidente, los procesos decisorios aparecen continuamente asociados al contexto económico y empresarial. Es frecuente encontrarnos con situaciones de incertidumbre en las que se debe escoger entre una pluralidad de opciones que pueden conducir a diferentes consecuencias, positivas o negativas, como resultado de la posible decisión elegida (Rabadán, Cid y Galán, 2013).

El proceso de revisión realizado sigue una metodología en la que se busca identificar las orientaciones teóricas respecto a la toma de decisiones, condensando la literatura representativa sobre el evento de estudio en un período determinado, lo cual generó 


\section{Gerencia de las organizaciones.}

Un enfoque empresarial

como resultado un sumario que evidencia las distintas formas de concebir el concepto. Posteriormente se presenta el resultado de la revisión efectuada. Se muestra el análisis de las investigaciones que sobre el tema han realizado las comunidades científicas académicas del país y del mundo, clasificadas en teóricos y empíricos. En la discusión y en las reflexiones se deja ver que desde el año 2000 apenas comienzan a tener espacio entre los investigadores los estudios sobre toma de decisiones en las revistas de más alto impacto.

\section{ESTADO DEL ARTE Y FUNDAMENTACIÓN TEÓRICA. APROXIMACIÓN AL CONCEPTO DE DECISIÓN}

Respecto a la indagación sobre el origen etimológico de la palabra decisión, hay que decir que proviene del latín decisio, deci-

252 sionis, el cual significa una opción seleccionada entre otras; más específicamente, el prefijo de, señala separación, caedere, significa cortar, talar, y el sufijo sión, que indica acción (Definición y etimología, 2017). De aquí se entiende que decidir implique seleccionar entre muchas opciones.

En el Diccionario de la Real Academia Española se define decisión con dos sentidos: primero como la determinación, resolución que se toma o se da en una cosa dudosa, y segundo, como la firmeza de carácter (2001, p.733). Por su parte, en el Diccionario General Etimológico de la Lengua Española se define decisión como determinación, resolución que se toma ó se da en alguna cosa dudosa (1887, p.629). De otro lado, para Islas (2001), la palabra decisión, según sus raíces griegas, proviene de krisis, del verbo krinein que significa separar, juzgar, decidir. 
Finalmente, en el diccionario de Ultimate Business de Cambridge, una decisión se define como: una elección que haces sobre algo después de pensar en varias posibilidades (Cambridge University Press, 2017). Según Rodríguez y Pinto (2010), han sido numerosos los investigadores que comenzaron a estudiar y profundizar sobre los componentes y principales características del proceso de decisión y su comportamiento en las organizaciones, entre ellos se encuentran Simon, March, Mintzberg, Manis, Moody, Huber, Choo y Pomerol. Quizás el de mayor prestigio de los mencionados, es Herbert A. Simon por sus aportes desde la economía, la administración y la psicología, con investigaciones orientadas hacia la toma de decisiones en las organizaciones empresariales.

Partiendo de lo anterior y teniendo en cuenta la aclaración etimológica y las definiciones de los diccionarios consultados, se entra de lleno al objetivo de indagar sobre las definiciones de la palabra decisión en los libros de texto. Dentro de la multitud de libros existentes, se seleccionaron algunos que siguen teniendo vigencia. Las definiciones encontradas en los diferentes textos consultados (véase Cuadro 1) tienen una postura homogénea sobre la definición.

En las definiciones presentadas se pueden identificar con claridad tres elementos usados para explicar la toma de decisión:

- Concepción de proceso.

- Elección de curso de acción.

- Solución de problemas o situaciones de oportunidad organizacional. 


\section{Gerencia de las organizaciones.}

Un enfoque empresarial

\section{Cuadro 1 \\ Definiciones de decisión y de toma de decisiones}

\begin{tabular}{l|l}
\hline Autor & Definición \\
\hline (Estrada, 2006) & $\begin{array}{l}\text { Para Herbert A. Simon la gerencia es sinónimo de } \\
\text { toma de decisiones, pero su principal interés fue } \\
\text { poner énfasis en el "cómo" (p.146) }\end{array}$ \\
(George, 1999) & $\begin{array}{l}\text { "La toma de decisiones es un proceso en el cual } \\
\text { todo el mundo ha adquirido mucha experiencia... } \\
\text { gran parte de esa experiencia ha tenido bastante } \\
\text { exito, por lo menos el suficiente para conservarnos } \\
\text { vivos y habernos traído a la situación actual" (p.17). }\end{array}$ \\
(Stephen, 2004) & $\begin{array}{l}\text { Las decisiones son "elecciones hechas entre dos o } \\
\text { más alternativas" (p.83). }\end{array}$
\end{tabular}

(Aktouf, 2001)

"El proceso por el cual se llega a una opción, pero una opción supuestamente aclarada, informada y motivada. Se trata de elegir entre varias formas posibles de actuar con miras a lograr una meta en condiciones y circunstancias dadas" (p.17).

(Pavesi, 2004)

"La decisión consiste en el proceso deliberado (y deliberativo) que lleva a la selección de una acción entre un conjunto de acciones alternativas. La decisión es un proceso previo a la acción" (p.12).

(Kast \& Rosenzweig,

"Adoptar una posición. Implica dos o más alternativas bajo consideración y la persona que decide tendrá que elegir entre ellas" (p.30).

(Moody, 1991)

"Una acción que debe tomarse cuando ya no haya más tiempo para recoger información" (p.29).

(Jones \& George, 2009)

"Proceso mediante el cual los gerentes responden a las oportunidades y amenazas que se les presentan, analizando las opciones y tomando determinaciones, o decisiones, relacionadas con las metas y líneas de acción organizacionales" (p.25).

(Manley, 1975)

"Curso de acción, elegido por quien decide, como el medio más efectivo disponible para alcanzar el objetivo u objetivos en los que actualmente está haciendo énfasis para resolver el problema que le preocupa" (p.62).

(Chiavenato, 2009) "La toma de decisiones es un proceso que consiste en hacer un análisis y elegir entre varias opciones un curso de acción" (p.36).

(Dougherty \& Pfaltzgraff, 1993)

"La toma de decisiones simplemente es el acto de elegir entre alternativas posibles sobre las cuales existe incertidumbre" (p.19).

Richard Daft, citado por

"La toma de decisiones organizacionales se defi(Almaraz, 2007) ne formalmente como el proceso de identificar y resolver problemas" (p.25).

Fuente: Elaboración propia (2016), a partir de diversos autores. 
Partiendo de lo anterior, Rodríguez (2014) afirma que la toma de decisiones organizacionales constituye un proceso informacional que se desarrolla por individuos o grupos para solucionar problemas y aprovechar oportunidades organizacionales. Por lo tanto, esta situación ratifica que la persona que toma decisiones al interior de las organizaciones empresariales, lo hace buscando la forma de mejorar el desempeño organizacional en beneficio de los distintos grupos de interés.

\section{INVESTIGACIONES SOBRE TOMA DE DECISIONES}

Con el objetivo de explorar los estudios sobre toma de decisiones en Colombia, se realizó una revisión sistemática en bases de datos especializadas, a partir de categorías como decisión, toma de decisiones, proceso de decisión y decisiones gerenciales, y se establecieron los siguientes límites: artículos publicados en revistas indexadas y publicaciones (empíricas y teóricas), así como tesis de universidades cuya temática fuera aplicable al contexto.

Finalmente se encontraron 60 documentos que se relacionaban de forma directa con las categorías planteadas. En la búsqueda emergieron estudios empíricos y académicos que han abordado la temática de la toma de decisiones y su dinámica de funcionamiento en las organizaciones. Lo cierto es que estos estudios han contribuido a una mayor y mejor comprensión del proceso de decisión en los entornos de negocios.

\section{MÉTODO}

En términos metodológicos, se pretende revisar las orientaciones teóricas respecto a la toma de decisiones, condensando la literatura representativa sobre el evento de estudio en un pe- 


\section{Gerencia de las organizaciones. \\ Un enfoque empresarial}

ríodo dado. Por tanto, el presente trabajo es una investigación teórico-descriptiva de tipo documental, dado que el procedimiento implica la búsqueda, organización y análisis de un conjunto de documentos físicos y electrónicos (ver Figura 1), sobre el tema de toma de decisiones.

La revisión documental se realizó en libros de texto y revistas de alto impacto, publicados en las bases de datos SCOPUS, SCIENCE DIRECT, EBSCO, SciELO y REDALYC.

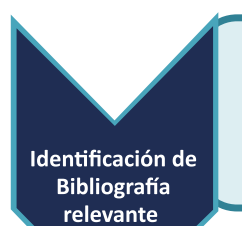

- Objetivo: Abarcar los conceptos de mayor importancia desarrollados respecto a la toma de decisiones.

- Actividades: Seleccionar libros de textos y revistas de mayor impacto/

Analizar la bibliografía/Hacer análisis de contenido.

Bibliografía

relevante

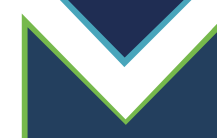

- Objetivo: Identificar las relaciones mediante un análisis de contenido.

- Actividades: Realizar fichas para cada libro de texto y para cada artículo

Generación de

científico/Identificar agrupaciones temáticas.

áreas temáticas

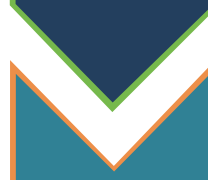

Interrelación

entre los

documentos
- Objetivo: Desarrollar relaciones entre las áreas temáticas.

- Actividades: Relacionar las distintas áreas temáticas/Hacer conclusiones sobre la evolución de la toma de decisiones.

\section{Figura 1}

Proceso estructurado de revisión bibliográfica

Fuente: Arévalo y Estrada (2016), a partir de Romero, Matamoros y Campo (2013)

\section{RESULTADO, DISCUSIÓN Y CONCLUSIÓN}

Los resultados de esta revisión se presentan a través de secciones. En la primera de ellas, mediante el uso de cuadros se exponen las investigaciones sobre toma de decisiones de las que se 
ha podido tener conocimiento tras cotejar las fuentes señaladas; por un lado, los documentos teóricos hallados, y por el otro, se incorporaron las investigaciones empíricas, y en cada uno de ellos se exponen las principales características descriptivas de los estudios seleccionados para la revisión. En la segunda, se presenta una discusión y reflexiones para el debate con base en los distintos estudios.

A continuación se recogen las investigaciones de las que se ha podido tener conocimiento tras revisar las fuentes señaladas. En el Cuadro 2 se presentan los documentos teóricos encontrados, con sus autores y títulos.

\section{Características descriptivas de las investigaciones teóricas}

En el trabajo de Céspedes (2009), se realiza una exposición breve del punto de vista filosófico de la teoría de la decisión, profundizando específicamente en el trabajo que propone David Lewis al respecto, quien sustenta que dicha teoría puede sostenerse sobre bases causales o no causales, siendo la Teoría Causal de la Decisión un modelo necesario para el análisis de este tipo de problemas. El artículo de Rodríguez y Pinto (2010) profundiza sobre los elementos teóricos asociados al proceso de toma de decisiones en las organizaciones, dígase su origen y evolución, así como su marcada importancia en los contextos organizacionales.

De igual forma, abordan las principales características de este proceso, enfatizando en sus componentes: situación problémica, decidor, información y contexto. Solano (2012) presenta los aspectos generales sobre la toma de decisiones enfocando el concepto de circuito de las decisiones; luego se discute cómo se 


\section{Gerencia de las organizaciones. Un enfoque empresarial}

\section{Cuadro 2 \\ Documentos teóricos revisados}

\begin{tabular}{|c|c|c|}
\hline No. & Autores & Título \\
\hline 1. & Esteban Céspedes (2009) & $\begin{array}{l}\text { La teoría de la decisión de David Lewis } \\
\text { y la paradoja de Newcomb. }\end{array}$ \\
\hline 2. & $\begin{array}{l}\text { Yunier Rodríguez Cruz; María Pinto Moli- } \\
\text { na (2010) }\end{array}$ & $\begin{array}{l}\text { Evolución, particularidades y carácter } \\
\text { informacional de la toma de decisiones } \\
\text { organizacionales. }\end{array}$ \\
\hline 3. & Solano, Ana Isabel (2012) & Toma de decisiones gerenciales. \\
\hline 4. & Doménec Melé (2000) & $\begin{array}{l}\text { Racionalidad ética en las decisiones } \\
\text { empresariales. }\end{array}$ \\
\hline 5. & Fernando Adolfo Fierro Celis (2014) & $\begin{array}{l}\text { Errores comunes en la toma de decisio- } \\
\text { nes estratégicas, un enfoque desde la } \\
\text { racionalidad. }\end{array}$ \\
\hline 6. & $\begin{array}{l}\text { Konstantinos V. Katsikopoulos; Cher- } \\
\text { ng-Horng (Dan) Lan (2011) }\end{array}$ & $\begin{array}{l}\text { Herbert Simon's spellon judgment and } \\
\text { decision making. }\end{array}$ \\
\hline 7. & $\begin{array}{l}\text { Gabriela Fernández Barberis, } \text { Ma del Car- }^{\mathrm{a}} \text { men Escribano Ródenas (2010) }\end{array}$ & $\begin{array}{l}\text { La ayuda a la decisión multicriterio: } \\
\text { orígenes, evolución y situación actual. }\end{array}$ \\
\hline 8. & $\begin{array}{l}\text { Patricia Gutiérrez Moreno, Martin Miguel } \\
\text { López García, Ramiro Torres Torres } \\
\text { (2013) }\end{array}$ & $\begin{array}{l}\text { Análisis de los riesgos en la toma de } \\
\text { decisiones de la alta dirección a través } \\
\text { de la auditoría de gestión. }\end{array}$ \\
\hline 9. & $\begin{array}{l}\text { Milko Ramsés González y Richard Obuchi } \\
\text { (2010) }\end{array}$ & $\begin{array}{l}\text { La ciencia, el arte y la psicología de } \\
\text { decidir. }\end{array}$ \\
\hline 10. & $\begin{array}{l}\text { Kennth Brousseau, Michael Dirver, Gary } \\
\text { Hourihan y RickardLarsson (2006) }\end{array}$ & $\begin{array}{l}\text { Estilos de toma de decisiones del ejecu- } \\
\text { tivo experimentado. }\end{array}$ \\
\hline 11. & Juan C. Cachanosky (2000) & $\begin{array}{l}\text { Las decisiones empresariales y las } \\
\text { predicciones en economía. }\end{array}$ \\
\hline 12. & $\begin{array}{l}\text { Onésimo Hernández-Lerma; Francisco } \\
\text { Venegas-Martínez (2012) }\end{array}$ & $\begin{array}{l}\text { Toma de decisiones de agentes raciona- } \\
\text { les con procesos Markovianos. }\end{array}$ \\
\hline 13. & Antonio Argandoña (2011) & $\begin{array}{l}\text { La ética y la toma de decisiones en la } \\
\text { empresa. }\end{array}$ \\
\hline 14. & $\begin{array}{l}\text { Ernesto Blanco Martínez y Milko González } \\
\text { (2007) }\end{array}$ & Las ventajas de decidir en grupo. \\
\hline 15. & Fabiola Baltar, Natacha Gentile (2012) & $\begin{array}{l}\text { Métodos mixtos para el estudio de las } \\
\text { decisiones estratégicas en las pymes. }\end{array}$ \\
\hline 16. & $\begin{array}{l}\text { Florina Guadalupe Arrendondo Trapero; } \\
\text { José Carlos Vázquez Parra ( } 2013\end{array}$ & $\begin{array}{l}\text { Un modelo de análisis racional para la } \\
\text { toma de decisiones gerenciales, desde } \\
\text { la perspectiva elsteriana. }\end{array}$ \\
\hline 17. & María Isabel Vélez Evans (2007) & $\begin{array}{l}\text { Aprender significa "perfeccionarse } \\
\text { siguiendo un camino". El proceso de } \\
\text { toma de decisiones estratégicas y el } \\
\text { aprendizaje organizacional. }\end{array}$ \\
\hline 18. & Javier L. Cristiano (2006) & $\begin{array}{l}\text { Racionalidad de la acción y racionali- } \\
\text { dad de la teoría. }\end{array}$ \\
\hline 19. & $\begin{array}{l}\text { Jaime Andrés Castañeda, Santiago Aran- } \\
\text { go, Yris Olaya (2009) }\end{array}$ & $\begin{array}{l}\text { Economía experimental en la toma de } \\
\text { decisiones en ambientes dinámicos y } \\
\text { complejos: Una revisión de diseños y } \\
\text { resultados. }\end{array}$ \\
\hline 20. & $\begin{array}{l}\text { Ignacio Loy Madera, Susana Carnero Sie- } \\
\text { rra, Félix Acebes Andreu,Patricia Solar } \\
\text { Peña, Ismael Âlvarez Bernardoy Joaquín } \\
\text { Morís Fernández (2009) }\end{array}$ & $\begin{array}{l}\text { La toma de decisiones como proceso } \\
\text { psicológico general: la supuesta excep- } \\
\text { ción de la psicología experimental del } \\
\text { condicionamiento. }\end{array}$ \\
\hline
\end{tabular}

Fuente: Elaboración propia (2016) 
relaciona la importancia de la decisión con la velocidad con que se procede a lo largo de este circuito, y los factores que diferencian una decisión importante de una de rutina. Del mismo modo aborda la relación entre el costo de recoger información y el beneficio derivado de esta información adicional.

Melé (2000) revisa críticamente algunos enfoques actuales en relación con el papel de la ética en la decisión, incluyendo la insuficiencia de los sentimientos, la tesis de separación, -que considera que hay decisiones puramente económicas y otras solo éticas - y la aplicación de éticas de tercera persona en la toma de decisiones. Como alternativa se defiende la necesidad de la racionalidad ética en todas las decisiones y la validez de un enfoque neoclásico de la racionalidad humana, de raíces aristotélicas. Fierro (2104) se aproxima a los conceptos de naturaleza de las decisiones, racionalidad, el proceso complejo de la toma de decisiones, el poder de la decisión y los modelos matemáticos.

El trabajo de Katsikopoulos y Cherng (2011), consistió en analizar 377 artículos científicos publicados en el Journal of Behavior en los cuales 91 de ellos citan a Simon, demuestran que muchos investigadores de la temática de decisiones se basan en la teoría de Herbert. Las investigadoras Fernández y Escribano (2010) se centran en la llamada Ayuda a la Decisión Multicriterio (Multiple Criteria Decision Aid), destacando dos aspectos fundamentales que dejan vislumbrar el término: ayuda, para indicar el cambio de orientación desde la llamada Toma de Decisiones Multicriterio. En su artículo Gutiérrez, López y Torres (2013) presentan unas reflexiones acerca de los beneficios de la aplicación de la auditoría de gestión, generando con ello la posibilidad de que 


\section{Gerencia de las organizaciones.}

Un enfoque empresarial

esta sea considerada por los empresarios como una herramienta que a futuro podría ahorrarles decisiones costosas.

González y Obuchi (2010) aducen que decidir es una función esencial del gerente y de cualquier ser vivo para garantizar su supervivencia. A su vez, afirman que en el estudio de las decisiones confluyen diversas disciplinas, desde la matemática hasta la psicología, y se encuentran diversos enfoques, desde el normativo (cómo se debe decidir), hasta el descriptivo (cómo decide la gente). Brousseau, Dirver y Hourihan (2006) crearon una matriz que identifica cuatro estilos de toma de decisiones: el decisivo (poca información, un curso de acción), el flexible (poca información, muchas alternativas), el jerárquico (mucha información, un curso de acción) y el integrador (mucha información, muchas alternativas).

En el artículo titulado Las decisiones empresariales y las predicciones en economía Cachanosky (2000) afirma que las decisiones correctas no implican resultados exitosos. Sin embargo, sistematizar los pasos de las predicciones para obligarse a explicitar los supuestos, ayuda a tener conciencia de las fortalezas y debilidades de las proyecciones. Concluye al final que no hay reglas para predecir.

En el estudio de Hernández y Venegas (2012) se revisa la evolución teórica y práctica de los procesos markovianos y resaltan su rápido avance y notorio potencial en el modelado de los procesos de toma de decisiones de agentes racionales. Dichos procesos han incorporado dinámicas más realistas en el comportamiento de diversas variables económicas y financieras que enriquecen el análisis en ambientes con riesgo e incertidumbre. Argandoña 
(2011) defiende la postura de que la ética es necesaria en la empresa porque forma parte de todas y cada una de las decisiones humanas. Las personas que participan en una empresa buscan resultados distintos y actúan por motivaciones variadas; por tanto, los directivos tienen que tener en cuenta varias dimensiones de sus acciones, que incluyen los beneficios materiales, pero también variables psicológicas, sociales y éticas.

Por su parte, Blanco y González (2007) indican que los procesos de decisión en grupo o participativos ofrecen grandes ventajas, comparados con las decisiones individuales. Sin embargo, aducen que este método no puede utilizarse en todas las situaciones ni con todos los grupos. Un proceso colectivo de decisión implica manejar diferentes opiniones y puntos de vista, analizar gran cantidad de información y utilizar sistemas para procesarla.

El objetivo del artículo de Baltar y Gentile (2012) es presentar una revisión teórica y metodológica de los estudios publicados en el ámbito de las decisiones estratégicas en las pymes, y presentar a modo ilustrativo, un diseño metodológico integral que permita caracterizar los perfiles decisorios y las prácticas observadas en este tipo de empresas. Arredondo y Vázquez (2013), con base en la perspectiva elsteriana (modelo racional para la toma de decisiones gerenciales, RTDG), presentan un modelo de análisis que propone como eje central la racionalidad en la toma de este tipo de decisiones. El modelo de análisis integra deseos, creencias y preferencias como los elementos que intervienen en la decisión del agente. Vélez (2007) ilustra en su artículo cómo es posible vincular el concepto de aprendizaje de Peter Senge con la idea de acción-decisión de Alfred Schutz y Darío Parra. Las inferencias presentadas argumentan por qué el proceso de toma 


\section{Gerencia de las organizaciones.}

Un enfoque empresarial

de decisiones puede ser considerado como un espacio para el aprendizaje, en tanto transforma las experiencias obtenidas en un cambio de actitud del decisor/directivo.

La investigación de Cristiano (2006) deja ver un análisis crítico de los cuatro argumentos centrales con que la teoría de la elección racional se postula como instrumento metodológico privilegiado de la sociología: el argumento de la «presunción razonable» esbozado por Elster, el del «privilegio paradigmático» de Abell, el del «marco integrador» de Van Parijs y el de la «fortaleza explicativa» de Freedman y Becker.

El documento de Castañeda y Arango (2009) revisa el uso de los experimentos de laboratorio en la economía, la psicología y las ciencias de la administración en el estudio de la toma de decisio-

262 nes en ambientes dinámicos y complejos. Tales ambientes no se han utilizado ampliamente en el laboratorio, aun sabiendo que mejoran la validación externa de los experimentos.

Finalmente, Loy, Cernero, Acebes y Solar (2009) proponen combativamente que el concepto de decisión se sitúe a la altura de los conceptos de asociación, conducta o representación, como alternativas al proceso psicológico esencial. Expresan que en todos los ámbitos experimentales de la psicología se ha producido una evolución conceptual hacia teorías basadas en algún tipo de concepto de decisión. Estos autores han defendido que el éxito de una concepción decisoria de la sensibilidad, la percepción, la memoria y el pensamiento, depende del momento en que se produzca.

En el Cuadro 3 se presentan los documentos empíricos encontrados, con sus autores y títulos. 


\section{Cuadro 3 \\ Documentos empíricos revisados}

\begin{tabular}{|c|c|c|}
\hline No. & Autores & Título \\
\hline 1. & $\begin{array}{l}\text { Leonor Cabeza de Vergara, Alber- } \\
\text { to Elías Muñoz Santiago y Sandra } \\
\text { Milena Vivero Santis (2004) }\end{array}$ & $\begin{array}{l}\text { Aproximación al proceso de toma de decisiones } \\
\text { en la empresa barranquillera. }\end{array}$ \\
\hline 2. & $\begin{array}{l}\text { Patricia González González y Ta- } \\
\text { tiana Bermúdez Rodríguez ( } 2008 \text { ) }\end{array}$ & $\begin{array}{l}\text { Una aproximación al modelo de toma de de- } \\
\text { cisiones usado por los gerentes de las micro, } \\
\text { pequeñas y medianas empresas ubicadas en Cali, } \\
\text { Colombia, desde un enfoque de modelos de deci- } \\
\text { sión e indicadores financieros y no financieros. }\end{array}$ \\
\hline 3. & María Isabel Vélez Evans (2006) & $\begin{array}{l}\text { El proceso de toma de decisiones como un espa- } \\
\text { cio para el aprendizaje en las organizaciones. }\end{array}$ \\
\hline 4. & $\begin{array}{l}\text { Iván Darío Ruiz Rodríguez y Jairo } \\
\text { Hernán Abello Plata (2012) }\end{array}$ & $\begin{array}{l}\text { La intuición estratégica como eje dinamizador en } \\
\text { la toma de decisiones de las mipymes. }\end{array}$ \\
\hline 5. & $\begin{array}{l}\text { María Elizabeth Castillo de Ma- } \\
\text { theus (2010) }\end{array}$ & Empresarios exitosos: cómo toman decisiones. \\
\hline 6. & Rodrigo Posada Bernal (2007) & $\begin{array}{l}\text { La toma de decisiones basada en los modelos de } \\
\text { investigación de operaciones en algunas empre- } \\
\text { sas industriales del sector agropecuario. }\end{array}$ \\
\hline 7. & $\begin{array}{l}\text { Francisco Ernesto Navarrete Báez, } \\
\text { Gabriel Hernández Rodríguez y } \\
\text { René Abbadie Morales (2012) }\end{array}$ & $\begin{array}{l}\text { La toma de decisiones en las micro, pequeñas } \\
\text { y medianas empresas de Jalisco: un proceso de } \\
\text { cambio basado en su cultura organizacional. }\end{array}$ \\
\hline 8. & $\begin{array}{l}\text { Jorge Eduardo Montoya López } \\
(2005)\end{array}$ & $\begin{array}{l}\text { Información en el proceso de toma de decisiones } \\
\text { estratégicas en las empresas colombianas del } \\
\text { grupo empresarial ISA. }\end{array}$ \\
\hline
\end{tabular}
Leandro Lepratte; Rafael Blanco;

9. Rubén Pietrobon; Diego Szlechter; Claudio Fardelli (2010)

El proceso de toma de decisiones estratégicas en pymes exitosas. Estudio de casos en Argentina.

10. Jorge Mario Cortés Cortés (2015)

11. Rosario Joaquín Reales Vega; Hilda Estrada López (2012)

Heurísticas y toma de decisiones gerenciales individuales en pymes de Bogotá.

La toma de decisiones en las pequeñas organizaciones del sector cooperativo y estilos de dirección.

Karla Ramírez-Barón, Omaira

12. Cecilia Martínez Moreno, María Habilidades sociales, factor determinante en la Concepción Ramírez Barón (2012)

13. Alberto Muñoz Santiago (2010) toma de decisiones en el sector servicios: restau) rantes turísticos, en Ensenada, B. C.

Análisis del proceso de toma de decisiones financieras en las empresas de Barranquilla desde la perspectiva del desarrollo moral.

Análisis del proceso de toma de decisiones en las grandes empresas de Barranquilla utilizando el análisis por conglomerados.

14. Leonor Cabeza de Vergara, Alber to Muñoz Santiago (2006)

Decisiones estratégicas a nivel competitivo y la

15. Rodrigo Alejandro Olivares Contreras (2013) influencia del género: estudio de la mujer empresaria en España.

16. Leonor Cabeza de Vergara, AlberAnálisis del proceso de toma de decisiones, visión desde la pyme y la gran empresa de Barranquilla.

17. Claudia P, Mendieta (2005)

La toma de decisiones en el sector público. Una mirada desde el dirigente.

18. Patricia González, Tatiana BermúFuentes de información, indicadores y herramientas más usadas por gerentes de mipyme en Cali, Colombia. 19. Yoannia Arean Rodríguez, Alejan- dro Rosete Suárez, Franklin Marín Evación de un modelo matemático para apodro Rosete Suárez, Franklin Marín yar decisiones empresariales complejas.
Vargas (2014)

Fuente: Elaboración propia (2016) 


\section{Gerencia de las organizaciones.}

Un enfoque empresarial

\section{Características descriptivas de las investigaciones empíricas} El estudio realizado por Cabeza, Muñoz y Vivero (2004) trata de analizar cómo se desarrolla el proceso de toma de decisiones en las empresas barranquilleras. Tomó como referencia una muestra de 77 empresas de la ciudad, en las cuales se percibe una alta tendencia a utilizar la intuición y la experiencia del decisor como herramientas fundamentales para resolver problemas. En los resultados se nota la necesidad de contar con modelos de simulación que contribuyan o aporten información que les permita tener más certeza a la hora de tomar una decisión ante una situación problemática.

La investigación de González y Bermúdez (2008) presenta los resultados de un estudio que pretende identificar el modelo de decisión, los indicadores y las herramientas gerenciales, que son usados por los gerentes de las mipymes ubicadas en Cali-Colombia. Concluyen que el modelo de decisión más utilizado por los gerentes es el racional, pues en un gran porcentaje, tienen claros los objetivos a alcanzar y los medios para lograrlos. El estudio dejó ver que las decisiones se toman por los gerentes de manera concertada con su comité o a través de reuniones participativas, identificándose así un modelo político.

En el trabajo de Vélez (2006) se considera el proceso de toma de decisiones como un espacio para el aprendizaje organizacional. Para dicho propósito se realizaron entrevistas semiestructuradas a un grupo de directivos de grandes empresas colombianas, para intentar dar respuesta a tres preguntas de investigación: 1) ¿cómo se puede involucrar el tema de aprendizaje con la idea de acción-decisión?, 2) ¿se puede considerar la toma de decisiones 
como una forma de aprendizaje?, y 3) ¿qué idea de aprendizaje tiene un grupo de directivos de grandes y medianas empresas en Colombia y cómo efectivamente lo incorporan en su proceso de toma de decisiones?

La tesis de maestría de Ruiz y Abello (2012) plantea un análisis sobre la incidencia que tiene la intuición estratégica en el proceso de creación, organización y permanencia de las organizaciones caracterizadas por pertenecer a la categoría de micro, pequeña y mediana empresa (mipyme) en Bogotá-Colombia. Se concentran en ver cómo las empresas observadas que se inician y perduran en el tiempo, lo hacen sin tener los respectivos estudios sistemáticos de diagnóstico de su entorno, tales como mercadeo, capacidad instalada, análisis financiero, entre otros.

En su investigación, Castillo (2010) busca interpretar y comprender la estructura que subyace en el proceso de toma de decisiones de empresarios exitosos sin estudios universitarios, de forma particular en el contexto empresarial. Hicieron un estudio cualitativo, en el que concluyeron las implicaciones que tienen la experiencia y el aprendizaje en la conformación de habilidades de tipo analítica, práctica y creativa.

Por su parte, la investigación de Posada (2007) se constituye en un instrumento de reflexión sobre la aplicación de los modelos de investigación de operaciones en el proceso de toma de decisiones, pues al estar estos cimentados en procesos de optimización de recursos, crean esquemas de acción tan rigurosos e inmodificables, que las relaciones laborales pueden sufrir un deterioro significativo al percibir los empleados la implemen- 


\section{Gerencia de las organizaciones.}

Un enfoque empresarial

tación de dichos procesos como herramientas coercitivas que pueden lesionar su integridad personal. El autor concluye que es un hecho que los modelos de investigación de operaciones son utilizados por las empresas que se dedican a la actividad industrial como elementos de toma de decisiones; algunos de ellos se denominan modelos PERT/CPM (Program Evaluation Review Technique, Critical Path Method).

Con su investigación, Navarrete, Hernández y Abbadie (2012), pretenden mostrar, a través de un análisis estadístico inferencial, apoyado de la prueba estadística de Análisis de Varianza ANOVA, cómo se presenta la gestión del proceso de toma de decisiones en las micro, pequeñas y medianas empresas de la zona metropolitana de Guadalajara, Jalisco, y cómo esta es determinada en gran parte por la cultura organizacional que posee.

El documento empírico de Montoya (2005), tuvo como objetivo determinar el grado de apoyo ofrecido por la información electrónica al proceso de decisiones estratégicas rutinarias y especificas tomadas a partir del año 1990 para el conjunto de empresas colombianas del grupo empresarial ISA, medido por el uso de dicha información. Concluye que hay una tendencia generalizada, por parte de los entrevistados, a pensar que las decisiones estratégicas son tomadas con una porción ligeramente mayor de la información externa sobre la interna, y que la información estratégica usualmente se ubica en el entorno.

El estudio de Lepratte, Blanc, Pietroboni, Szlechter \& Fardelli (2010), tuvo como objetivo principal analizar el proceso estratégico de pequeñas y medianas empresas (pymes), centrándose en 
la articulación organizacional de los componentes explícitos y tácitos que sirven como guía para la toma de decisiones. Se partió del supuesto de que la mayoría de los análisis de la literatura especializada carecen de precisiones acerca de la naturaleza de la gestión estratégica en este tipo de firmas, y que el proceso estratégico en las pymes tiene ciertas especificidades que lo diferencian de los procesos de las grandes firmas.

Por su parte, Cortés (2015), en su estudio pretendió evidenciar el uso de heurísticas en la toma de decisiones gerenciales en pequeñas y medianas empresas de Bogotá, especialmente en decisiones individuales y en la heurística de anclaje y ajuste. Para ello se realizó una encuesta con método probabilístico a 3.988 gerentes, cuyos resultados evidenciaron el uso de recursos tradicionales (racionales) y también heurísticos, en el momento en que los gerentes deciden en pequeñas y medianas empresas.

El documento de Reales y Estrada (2012) consistió en identificar la articulación entre el conocimiento sobre modelos de planificación y estilos de dirección en los directivos de pequeñas organizaciones. Utilizaron el método etnográfico con el apoyo de técnicas de investigación como la entrevista, la observación y los grupos focales, aplicados a directivos de la organización. En conclusión, el estudio establece que las pequeñas organizaciones cooperativas, en lugar de adecuarse al rol limitado y meramente complementario que el sistema capitalista les asigna, están contribuyendo a crear un hombre nuevo, capaz de materializar a largo plazo un proyecto propio de realización humana y trascendente.

Las pretensiones de Ramírez, K., Martínez,O. y Ramirez, M. 


\section{Gerencia de las organizaciones.}

Un enfoque empresarial

(2012), estuvieron en analizar qué tan determinantes son las habilidades sociales (de comunicación, influencia, establecimiento de vínculos y empatía), respecto a la toma de decisiones, del modelo racional limitado, en el nivel directivo, en el sector servicios de Ensenada, Baja California, específicamente en los restaurantes turísticos. La investigación fue descriptiva, correlacional transversal, contempló una fase de investigación cuantitativa, complementada con la cualitativa que permitió obtener información para mejorar los procesos de gestión interna, gracias al desarrollo de estas habilidades.

Para Muñoz (2010), su preocupación estuvo en orientar el análisis de los resultados obtenidos en una investigación dirigida al personal encargado de la toma de decisiones financieras en diferentes empresas de Barranquilla, a partir de los fundamentos teóricos que existen sobre los juicios morales enunciados por Lawrence Kohlberg, con el fin de conocer las variables y valores que influyen en esta gente durante el proceso de decisión, desde una perspectiva moral.

El estudio de Cabeza y Muñoz (2006), muestra los resultados obtenidos en una investigación dirigida a identificar los modelos utilizados por los tomadores de decisión en las diversas áreas funcionales de la gran empresa barranquillera, tomando como herramienta básica el análisis de conglomerados y las variables empleadas en dicho proceso de decisión.

El documento empírico de Olivares (2013), intenta descubrir si mujeres y hombres poseen diferentes formulaciones estratégicas, analizando las estrategias competitivas de la diferenciación, 
la innovación y la expansión de mercados. Los resultados se obtuvieron a partir de una muestra de la encuesta a la población adulta (APS) del Global Entrepreneurship Monitor de España del 2009, aplicando un modelo logit. Se descubrió que no existen grandes diferencias en el comportamiento estratégico entre hombres y mujeres; únicamente se encontraron diferencias en la estrategia competitiva de la innovación, donde el hecho de ser mujer influye positivamente en su selección.

En otra investigación de Cabeza y Muñoz (2010), su afán estuvo en analizar cómo se desarrolla el proceso de toma de decisiones en las empresas barranquilleras, desde un punto de vista netamente descriptivo. Se tomó una muestra de 77 empresas pymes de la ciudad de Barranquilla, en las cuales se percibe una alta tendencia a utilizar la intuición y la experiencia del tomador de decisiones como herramientas fundamentales para resolver problemas. Palpándose, por las respuestas obtenidas, la necesidad de contar con modelos de simulación que contribuyan o aporten información que les permita tener más certeza a la hora de tomar un curso de acción ante una situación problemática.

Con su artículo, Mendieta (2005) deja ver su interés por contrastar aspectos de formación, creencias y aversión al riesgo de varios dirigentes vallecaucanos. Para lograrlo, recurrió a entrevistas con directivos gubernamentales y servidores públicos de trece municipios del Valle del Cauca, en los que se pudo concluir que, pese a existir una creciente ola de tecnocracia en la gestión pública colombiana, esta aún no se arraiga lo suficiente en los procesos de toma de decisiones de la región. 


\section{Gerencia de las organizaciones.}

Un enfoque empresarial

De otro lado, González y Bermúdez (2010) presentan los resultados de una investigación de carácter descriptivo que pretende identificar las fuentes de información, los indicadores y las herramientas gerenciales más utilizadas por gerentes de mipymes en Cali, Colombia. Para tal fin se realizó una encuesta a 270 gerentes de mipymes, cuyas empresas se encuentran inscritas en la Cámara de Comercio de ese país. Se observó que los gerentes para la toma de decisiones principalmente se apoyan en indicadores financieros, los cuales surgen de la combinación de indicadores, tanto de rentabilidad como de liquidez y endeudamiento.

Finalmente, el trabajo de investigación de Arean, Rosete y Marín (2014), consistió en diseñar un nuevo modelo matemático que respondiera a un caso particular del problema del viajante de comercio con ventanas de tiempo y la evaluación de la función objetivo obtenida, utilizando cinco algoritmos metaheurísticos.

La aproximación al tema de la toma de decisiones permite evidenciar que existen numerosos estudios al respecto. Se presenta a continuación un examen de los datos obtenidos en esta revisión bibliográfica. El objetivo de esta investigación ha sido analizar los avances científicos literarios sobre la toma de decisiones en el contexto empresarial mediante un estudio bibliométrico. En esta línea se puede decir que se consultaron en total 61 documentos, entre libros de texto, artículos científicos, tesis de maestría y doctorado, que se relacionaban de forma directa con las categorías planteadas.

En la primera sección se presenta una estructura de los conceptos de decisión y de toma de decisiones con la mirada de 
distintos autores, encontrándose homogeneidad en la forma de concebirlos. En términos generales, esto quiere decir que la percepción sobre la toma de decisiones mostrada en la literatura, da lugar a entender que las decisiones en una organización deben ser consideradas como un proceso que pretende la solución de problemas.

De acuerdo a la búsqueda realizada en bases de datos especializadas en los documentos teóricos y empíricos encontrados, se conoce sobre todo de estos últimos; que a partir del año 2000, es cuando realmente apenas comienzan a tener espacio entre los investigadores los estudios empíricos, entendidos estos, según Malaver (2000), citado por Freitas (2009), como aquellos que tienen por objeto de estudio un hecho o fenómeno de la calidad empresarial que es sometido a observación y análisis con el fin de entenderlo, y por esa vía poder contribuir a mejorar la gestión y desempeño de las organizaciones.

Entre los años 2001 y 2015 se identifica un avance en los trabajos. Se nota un crecimiento en cantidad y calidad de los estudios, a los cuales se han vinculado investigadores de alto prestigio de la comunidad académica nacional e internacional.

En síntesis, los trabajos son relativamente recientes y casi en proporciones iguales los de carácter teórico y empírico. De los documentos hallados hay que decir que han sido publicados en revistas científicas que denotan alta calidad, y que han sido listados en alguna base de datos de consulta mundial. Esto demuestra que el tema de la toma de decisiones ha sido de interés por la comunidad científica. 


\section{Gerencia de las organizaciones.}

Un enfoque empresarial

Por lo anterior se destaca el valor de abordar el tema de la toma de decisiones ya que Simon (1964) la considera el núcleo de la Administración, y que el vocabulario de la teoría de la Administración debe derivarse de la lógica y de la psicología de la elección humana. Toda acción abarca tanto la elección previa a dicha acción como al hacer que deriva de dicha elección (Doval, 2009). De acuerdo con la revisión efectuada, este trabajo agrega valor en términos académicos, ya que este escrito se constituye en una herramienta útil, que proporciona nuevas miradas conceptuales sobre la toma de decisiones en el contexto empresarial.

Para finalizar, se debe indicar que con este trabajo no concluye de manera definitiva el tema de la toma de decisiones, por tanto, al respecto conviene decir que, a pesar de todos los estudios recolectados, quedan preguntas sin contestar que necesitan clarifi-

272 carse; es así que los resultados obtenidos deben de ser tomados con cautela, ya que se dejaron por fuera aportes significativos a la teoría, expuestos en otros libros de texto y en otras revistas científicas.

\section{REFERENCIAS BIBLIOGRÁFICAS}

Aktouf, O. (2001). La administración: entre Tradición y Renovación. Cali: Artes Gráficas del Valle.

Almaraz, I. (2007). Análisis de los factores que intervienen en la toma de decisiones de los administradores dentro de las organizaciones. Querétaro: Universidad Autómoma de Querétaro. Arean, Y., Rosete, A. y Marín, F. (2014). Evaluación de un modelo matemático para apoyar decisiones empresariales complejas. Revista Internacional Administración y Finanzas, 7(6), 1-13.

Argandoña, A. (2011). La ética y la toma de decisiones en la empresa. Universia Business Review, 2do trimestre, 30, 1-11. 
Arredondo, F. y Vázquez, J. C. (2013). Un modelo de análisis racional para la toma de decisiones gerenciales, desde la perspectiva Elsteriana. Cuad. admon.ser.organ. 26(46), 135-158.

Baltar, F. y Gentile, N. (2012). Métodos mixtos para el estudio de las decisiones estratégicas en las pymes. Global Conference on Business and Finance Proceedings, 7(1), 1060-1071.

Blanco, E. y González, M. (2007). Las ventajas de decidir en grupo. Debates IESA, XII(4), 29-36.

Brousseau, K., Dirver, M. y Hourihan, G. (2006). Estilos de toma de decisiones del ejecutivo experimentado. Revista Iberoamericana de Ciencias Empresariales y Economía, 3(3), 1-5.

Cabeza, L. (2004). Aproximación al proceso de toma de decisiones en la empresa barranquillera. Pensamiento y Gestión, 2(17), 1-38.

Cabeza, L. y Muñoz, A. (2006). Análisis del proceso de toma de decisiones en las grandes empresas de Barranquilla utilizando el análisis por conglomerados. Pensamiento y Gestión, 1(20), 55-109.

Cabeza, L. y Muñoz, A. (2010). Análisis del proceso de toma de decisiones, visión desde la pyme y la gran empresa de Barranquilla. Cuadernos Latinoamericanos de Administración, 6(10), 9-40.

Cachanosky, J. (2000). Las decisiones empresariales y las predicciones en economía. Revista Libertas, 32(1), 1-23.

Cambridge University Press (febrero de 2017). Obtenido de http://dictionary.cambridge.org/es/diccionario/ingles/ ultimate

Castañeda, J. y Arango, S., Olaya, Y. (2009). Economía experimental en la toma de decisiones en ambientes dinámicos y complejos: Una revisión de diseños y resultados. Cuadernos de Administración, 22(39), 31-57. 


\section{Gerencia de las organizaciones.}

Un enfoque empresarial

Castillo, M. (2010). Empresarios exitosos: cómo toman decisiones. Revista Venezola de Gerencia, 15(52), 548-569.

Céspedes, E. (2009). La teoría de la decisión de David Lewis y la paradoja de Newcomb. Límite, 4(20), 53-68.

Chiavenato, I. (2009). Comportamiento organizacional. Bogotá: McGraw-Hill.

Cortés, J. (2015). Heurísticas y toma de decisiones gerenciales individuales en pymes de Bogotá. Bogotá: Universidad Nacional de Colombia.

Cristiano, J. (2006). Racionalidad de la acción y racionalidad de la teoría. Reis. Revista Española de Investigaciones Sociológicas, 1(113), 135-153.

De Echegaray, E. (1887). Dicccionario etimólogico de la lengua española. Madrid: Universidad de Toronto.

Definición y etimología (02 de febrero de 2017). Definición. Obtenido de https://definiciona.com/decision/

Dougherty, J. y Pfaltzgraff, R. (1993). Teorías en pugna en las relaciones internacionales. Buenos Aires: Grupo Editor Latinoamericano.

Doval, I. (2009). La ética en la toma de decisiones: la consideración de los valores mediante la acción comunicativa (Tesis Doctoral). Buenos Aires: Universidad de Buenos Aires.

Estrada, F. (2006). Herbert A. Simon y la economía organizacional. Revista Sociedad y Economía, 1(11), 146-174.

Fernández, G. y Escribano, M. (2010). La ayuda a la decisión multicriterio: orígenes, evolución y situación actual. Madrid: Universidad CEU San Pablo.

Fierro, F. (2104). Errores comunes en la toma de decisiones estratégicas, un enfoque desde la racionalidad. Revista de Estudios Avanzados de Liderazgo, 1(3), 78-100. 
Freitas, S. (2009). Investigación empírica en contabilidad de gestión, estrategia para el estudio de los costos de calidad en las organizaciones. Revista Venezolana de Análisis de Coyuntura, 15(2), 165-181.

George, H. (1999). Toma de decisiones en la gerencia. México: Trillas.

González, P. y Bermúdez, T. (2008). Una aproximación al modelo de toma de decisiones usado por los gerentes de las micro, pequeñas y medianas empresas ubicadas en Cali, Colombia desde un enfoque de modelos de decisión e indicadores financieros y no financieros. Cont. udea, 1(52), 131-154.

González, P. y Bermúdez, T. (2010). Fuentes de información, indicadores y herramientas más usadas por gerentes de Mipyme en Cali, Colombia. Contad. Adm, (232), 83-108.

González, M. y Obuchi, R. (2010). La ciencia, el arte y la psicología de decidir. Debates Iesa, 15(1), 23-26.

Gutiérrez, P., López, M. y Torres, R. (2013). Análisis de los riesgos en la toma de decisiones de la alta dirección a través de la auditoría de gestión. Global Conference on Business and Finance Proceedings, 8(1), 955-963.

Hernández, O. y Venegas, F. (2012). Toma de decisiones de agentes racionales con procesos Markovianos. El Trimestre Económico, LXXIX(316), 733-779.

Islas, L. (2001). El proceso de toma de decisiones racionales en el enfoque de políticas públicas. México: Universidad Autónoma Metropolitana.

Jones, G. y George, J. (2009). Administración Contemporánea.México: McGraw-Hill.

Kast, F. y Rosenzweig, J. (1998). Administración en las organizaciones. Un enfoque de sistemas y contingencias. México: McGraw-Hill. 


\section{Gerencia de las organizaciones.}

Un enfoque empresarial

Katsikopoulos, K. y Cherng, H. (2011). Herbert Simon's spell on judgment and decision making. Judgment and Decision Making, 6(8), 722-732.

Lepratte, L., Blanc, R., Pietroboni, R., Szlechter, D. y Fardelli, C. (2010). El proceso de toma de decisiones estratégicas en pymes exitosas. Estudio de casos en Argentina. Buenos Aires: Academia.edu

Loy, I., Cernero, S., Acebes, F. y Solar, P. (2009). La toma de decisiones como proceso psicológico general: la supuesta excepción de la psicología experimental del condicionamiento. Revista de Historia de la Psicología, 30(23), 195-206.

Manley, J. (1975). Las decisiones del ejecutivo. México: Ed. Continental.

Melé, D. (2000). Racionalidad ética en las decisiones empresariales. Revista Empresa y Humanismo, II(2), 411-437.

Mendieta, C. (2005). La toma de decisiones en el sector público. Una mirada desde el dirigente. Revista Científica Guillermo de Ockham, 3(2), 31-48.

Montoya, J. (2005). Información en el proceso de toma de decisiones estratégicas en las empresas colombianas del grupo empresarial ISA. Medellín: Universidad EAFIT.

Moody, P. (1991). Toma de decisiones gerenciales. México: McGraw-Hill.

Muñoz, A. (2010). Análisis del proceso de toma de decisiones financieras en las empresas de Barranquilla desde la perspectiva del desarrollo moral. Pensamiento y Gestión, 1(20), 54-84. Navarrete, F., Hernández, G. y Abbadie, R. (2012). La toma de decisiones en las micro, pequeñas y medianas empresas de Jalisco: un proceso de cambio basado en su Cultura Organizacional. Jalisco: Universidad del Valle de Atemajac (UNIVA). 
Olivares, R. (2013). Decisiones estratégicas a nivel competitivo y la influencia del género: estudio de la mujer empresaria en España. Pensamiento y Gestión, 1(34), 161-182.

Pavesi, L. (2004). La decisión. Buenos Aires: Norma.

Posada, R. (2007). La toma de decisiones basada en los modelos de investigación de operaciones en algunas empresas industriales del sector agropecuario. Medellín: Universidad EAFIT.

Rabadán, A., Cid, A. y Galán, S. (2013). Métodos de Decisión Empresarial. Madrid: Delta.

Ramírez, K., Omaira, M. y María, R. (2012). Habilidades sociales, factor determinante en la toma de decisiones en el sector servicios: restaurantes turísticos, en Ensenada, B. C. Global Conference on Business and Finance Proceedings, 7(1), 1098-1108. Real Academia Española (2001). Diccionario de la lengua española. Madrid: Espasa.

Reales, R. y Estrada, H. (2012). La toma de decisiones en las pequeñas organizaciones del sector cooperativo y estilos de dirección. Desarrollo Gerencial, 4(1), 20-52.

Rodríguez, Y. (2014). Modelo de uso de información para la toma de decisiones estratégicas en organizaciones de información cubanas. Granada: Editorial de la Universidad de Granada.

Rodríguez, Y. y Pinto, M. (2010). Evolución, particularidades y carácter informacional de la toma de decisiones organizacionales. Acimed, 21(1), 57-77.

Romero, J., Matamoros, S. y Campo, C. (2013). Sobre el cambio organizacional. Una revisión bibliográfica. Innovar, 23(50), 35-52.

Ruiz, I. y Abello, J. (2012). La intuición estratégica como eje dinamizador en la toma de decisiones de las mipymes (Tesis de Maestría). Bogotá: Universidad Nuestra Señora del Rosario. 


\section{Gerencia de las organizaciones.}

Un enfoque empresarial

Solano, A. (2012). Toma de decisiones gerenciales. Tecnología en Marcha, 16(3), 44-51.

Stephen, R. (2004). Comportamiento Organizacional. México: Pearson.

Simon, H. (1964). El comportamiento administrativo. México: Aguilar.

Vélez, M. (2006). El proceso de toma de decisiones como un espacio para el aprendizaje en las organizaciones. Revista Ciencias Estratégicas, 14(16), 153-159.

Vélez, M. (2007). Aprender significa "perfeccionarse siguiendo un camino". El proceso de toma de decisiones estratégicas y el aprendizaje organizacional. Semestre Económico, 10(19), 69-84.

\section{Cómo citar este capítulo:}

Arévalo-Ascanio, J., y Estrada López, H. (2017). La toma de decisiones. Una revisión del tema. En Prieto-Pulido, R. y García-Guiliany, J. (2017). Gerencia de las organizaciones. Un enfoque empresarial. Ediciones Universidad Simón Bolívar. Barranquilla-Colombia. 249-278. 